The Free Internet Journal for Organic Chemistry
Paper

Arkivoc 2019, part v, 10-18

Organic Chemistry

\title{
Synthesis and transformations of a few 9-(pent-4-yn-1-yl)anthracene-type systems
}

Eason M. Mathew, Tomson Devassia, Sreedharan Prathapan, and Perupparampil A. Unnikrishnan*

Department of Applied Chemistry, CUSAT, Kochi-22, India

Email:paunni@gmail.com

Received 05-24-2018

Accepted 11-04-2018

Published on line $12-27-2018$

\section{Abstract}

9-(Pent-4-yn-1-yl)anthracene-type compounds can potentially undergo intramolecular Diels-Alder (IMDA) reaction to form 9,11-annulated dibenzobarrelenes. Herein we report the synthesis and IMDA reactions of several heteroatom incorporated 9-(pent-4-yn-1-yl)anthracene-type compounds.
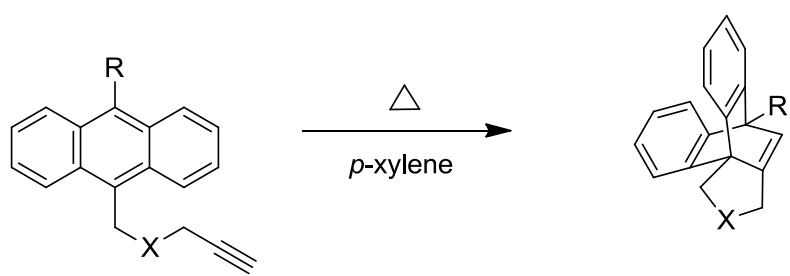

a) $\mathrm{R}=\mathrm{H}$ b) $\mathrm{R}=\mathrm{CH}_{3}$ c) $\mathrm{R}=\mathrm{OCH}_{3}$ d) $\mathrm{R}=\mathrm{Ph}$

b) $\mathrm{X}=\mathrm{S}, \mathrm{SO}_{2}, \mathrm{O}$

Keywords: Dibenzobarrelenes, Intramolecular Diels-Alder (IMDA) reaction, tethered barrelenes, fused-ether, ester, sulfide and sulfone, ${ }^{1} \mathrm{H}$ and ${ }^{13} \mathrm{C}$ NMR 


\section{Introduction}

Synthesis of bicyclo[2,2,2] octa-2,5,7-triene (barrelene) was first reported by Zimmerman ${ }^{1}$ et al in 1960 . Its barrel-shaped array of molecular orbitals and three ethylene units that are like staves attached to the two methine units attracted the attention of chemists. Synthesis of several barrelene derivatives, especially dibenzobarrelenes, exploited Diels-Alder reaction. ${ }^{2-7}$ Intramolecular Diels-Alder (IMDA) reaction of suitably substituted anthracenes to give tricyclic systems that may be regarded as annulated barrelenes was first reported by Meek and Dann. ${ }^{8,9}$ In 1980, Ciganek ${ }^{5}$ reported a systematic investigation on the synthesis of 9,11bridged dibenzobarrelene via IMDA reaction (Scheme 1). Entropically favoured IMDA reaction generally proceeded with increased reaction rates under mild reaction conditions and the products were obtained in good yield.

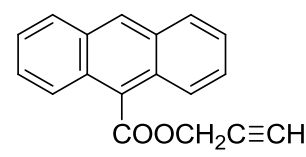

1

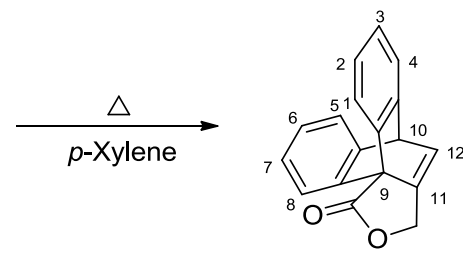

2

\section{Scheme 1}

Barrelene undergoes singlet mediated rearrangement to give cyclooctatetraene and triplet mediated di- $\pi$ methane rearrangement ${ }^{10-12}$ to afford semibullvalene. Initially, diverse photochemistry of dibenzobarrelenes occupied center stage and most attempts on dibenzobarrelene synthesis were directed towards deciphering the effect of substituents on controlling the photochemistry of barrelenes. ${ }^{13}$ However chemistry of barrelenes has now transcended to encompass several fields including recently found applications in OLEDs $^{14}$ and photoluminescent materials. ${ }^{15,16}$ Dibenzobarrelene based azaacenes found enhanced device performance than corresponding appended iptycene motifs. ${ }^{14}$ Dibenzobarrelenes have been exploited in the biological field also; dibenzobarrelene fused with thiazole and thiophene entities showbiological activities ${ }^{17}$ and are employed in drug discovery. In this context, we explored the possibility of synthesizing different types of 9,11-annulated barrelenes with ester, ether, sulfide and sulfone tethers constructed between 9- and 11-positions in the newly synthesized barrelenes.

\section{Result and Discussion}

We employed IMDA strategy to generate several 9,11-bridged dibenzobarrelenes where ester, sulfide, sulfone and ether linkages constituted the putative 9,11-bridges. As expected, IMDA reactions proceeded under mild conditions with high reaction rates. Structure of IMDA adducts were arrived at on the basis of spectral and analytical data and single crystal X-ray diffraction studies on a few representative examples. Structure of 9(pent-4-yn-1-yl)anthracene-type compounds $(3,4)$ and their IMDA products $(5,6,7,8)$ are listed in Figure 1. Steps involved in the synthesis of 9-(pent-4-yn-1-yl)anthracene-type compounds and their respective IMDA products are depicted in Scheme 2. 
<smiles>[R]c1c2ccccc2c(C(=O)OCC#C)c2ccccc12</smiles>

3<smiles>[X]CCc1c2ccccc2c([R])c2ccccc12</smiles>

$4 \quad X=O, S$

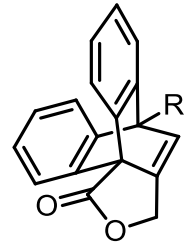

5

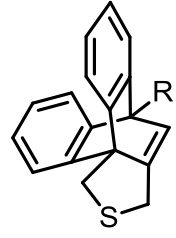

6

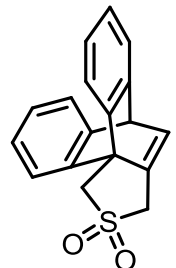

7

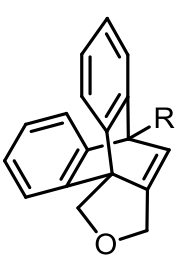

8

a) $\mathrm{R}=\mathrm{H}$ b) $\mathrm{R}=\mathrm{CH}_{3}$ c) $\mathrm{R}=\mathrm{OCH}_{3}$ d) $\mathrm{R}=\mathrm{Ph}$

Figure 1

Aldehydes 9 acted as common precursors for our targets. They could be oxidized with $t$-butylhydroperoxide (TBHP) in $t$-butanol to give the corresponding acids $\mathbf{1 0}$ in high yields. Acids $\mathbf{1 0}$ were quantitatively converted to the corresponding acid chlorides 12 by treating with cyanuric chloride $\mathbf{1 1}$. Reaction of 12 with propargyl alcohol gave the corresponding propargyl esters 13. IMDA reactions of 13 was successfully performed in refluxing in p-xylene to giver ester bridged dibenzobarrelenes 5a-d (Scheme 2). Structures of all synthesized bridged esters were confirmed by spectral and analytical data. Compound $\mathbf{5 a}{ }^{5}$ exhibited in ${ }^{1} \mathrm{H}$ NMR a signal at $\delta 6.75$, assigned to vinylic proton, whereas the bridgehead proton appeared as a doublet at $\delta 5.21$. The eight aromatic protons appeared as a multiplet in the $\delta 6.95-7.45$ region and the doublet due to two protons at $\delta 4.98$ was assigned to methylene protons. The ${ }^{1} \mathrm{H}$ NMR spectrum of $\mathbf{5 b}$ and $\mathbf{5 c}$ showed a singlet due to three protons at $\delta 2.23$ and $\delta 3.94$ respectively, assigned to methyl and methoxy protons. Single Crystal XRD (ORTEP diagrams) obtained for compounds $\mathbf{5} \mathbf{c}$ and $\mathbf{5} \mathbf{d}$ are given in the Figure 2.

Sulfide bridged barrelenes 6 were also synthesized from aldehydes 9, which after reduction with sodium borohydride/methanol gave corresponding alcohols 14. Reaction between alcohol $\mathbf{1 4}$ and two equivalents of thiourea in acetone in the presence of $5 \mathrm{~N} \mathrm{HCl}$ followed by treatment sodium hydroxide gave thiol 16. Anthracenethiols $\mathbf{1 6}$ dissolved in chloroform and $\mathrm{KOH}$ dissolved in methanol were mixed and stirred overnight followed by addition of propargyl bromide to generate propargyl sulfide $\mathbf{1 7}$ that underwent IMDA reaction in $p$-xylene to give bridged sulfides 6 (Scheme 2). Structure of bridged sulfides 6 were confirmed by analytical results and spectral data.

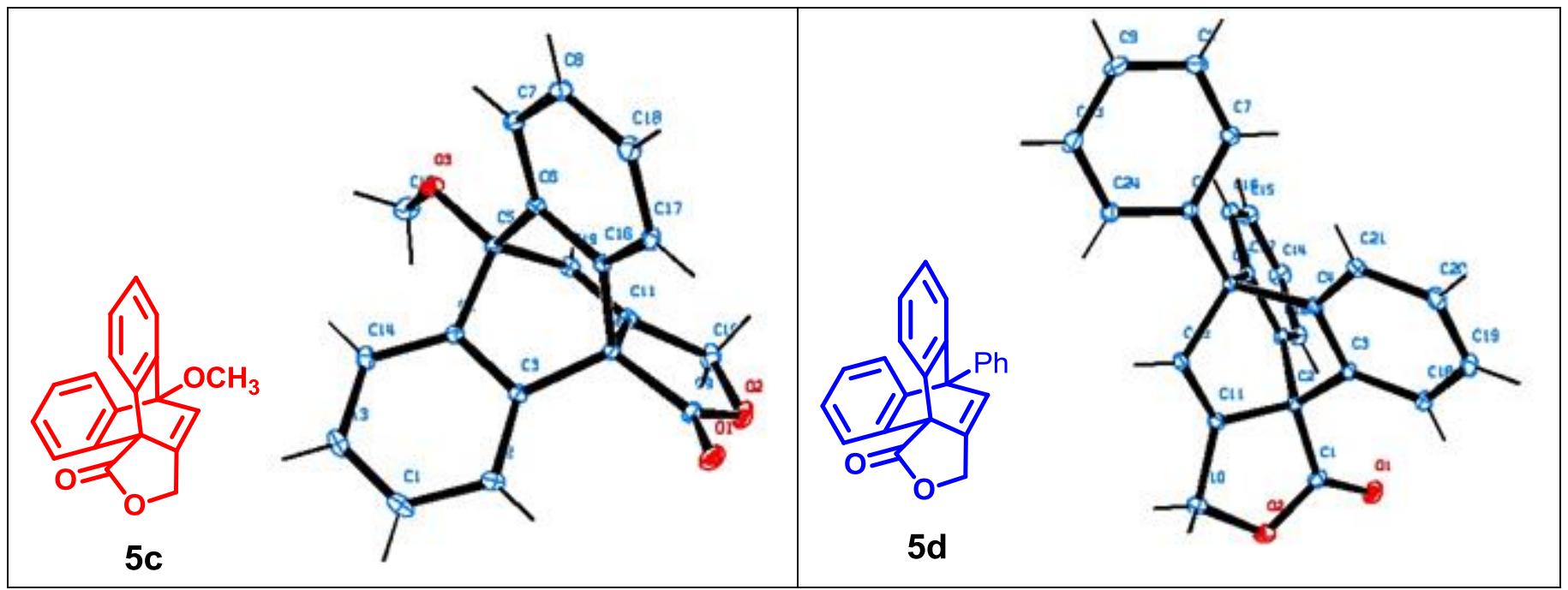

Figure 2 
Boric acid catalyzed reaction of bridged sulfide 6 a with $30 \%$ hydrogen peroxide (Scheme 2 ) resulted in the formation of corresponding bridged sulfone 7. Structure of bridged sulfone 7 was elucidated on the basis of analytical results and spectral data. In the IR spectrum, sulfones generally show strong absorption bands at $1350-1300 \mathrm{~cm}^{-1}$ region due to asymmetric $\mathrm{SO}_{2}$ stretching. The asymmetric $\mathrm{SO}_{2}$ stretching of 7 occurred at 1318 $\mathrm{cm}^{-1}$.

Sodium salt of anthracenemethanols 14 on reaction with propargyl bromide afforded the corresponding propargyl ethers 19. IMDA reaction of 19 in refluxing $p$-xylene gave ether bridged dibenzobarrelenes 8 (Scheme 2). Structures of bridged ethers $\mathbf{8}$ were also established on the basis of analytical results and spectral data.<smiles>[R]c1c2ccccc2c(C(=O)O)c2ccccc12</smiles><smiles>CCNC(=O)OC(C)(C)CO</smiles>

11<smiles>[R]c1c2ccccc2c(C(=O)Cl)c2ccccc12</smiles>

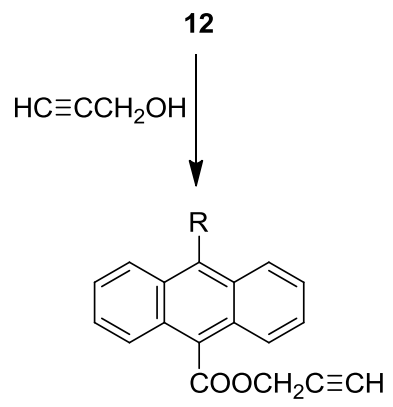

13<smiles>[R]c1c2ccccc2c(CO)c2ccccc12</smiles>

14<smiles>[R]c1c2ccccc2c(CS)c2ccccc12</smiles><smiles>[R]c1c2ccccc2c(C)c2ccccc12</smiles><smiles></smiles><smiles>[R]c1c2ccccc2c(C)c2ccccc12</smiles>

19

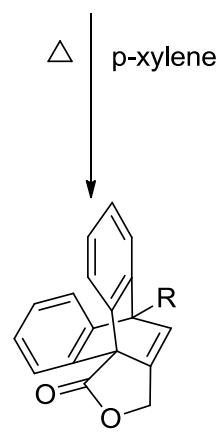

5<smiles>[R]c1c2ccccc2c(C)c2ccccc12</smiles><smiles></smiles>
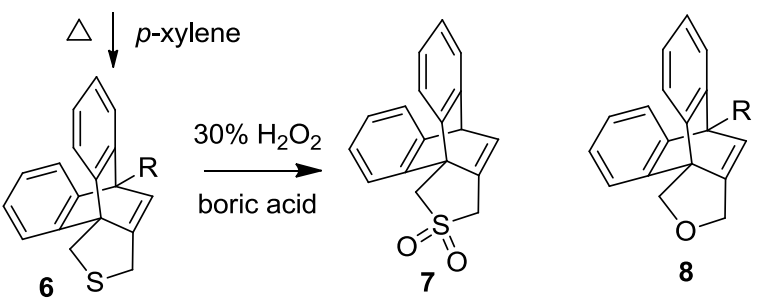

a) $\mathrm{R}=\mathrm{H}$ b) $\mathrm{R}=\mathrm{CH}_{3}$ c) $\mathrm{R}=\mathrm{OCH}_{3}$ d) $\mathrm{R}=\mathrm{Ph}$

\section{Scheme 2}




\section{Conclusions}

Several 9-(pent-4-yn-1-yl)anthracene-type compounds were successfully synthesized in high yields and were converted to the corresponding 9,11-bridged dibenzobarrelene derivatives in high yields via an entropically favored IMDA reaction. These bridged dibenzobarrelenes are potential candidates to examine regiochemical preferences in barrelene to semibullvalene rearrangement.

\section{Experimental Section}

General. Melting points are uncorrected and recorded on a Neolab melting point apparatus. Infrared spectra were recorded on Jasco 4100 and ABB Bomem (MB Series) FT-IR spectrometers. ${ }^{1} \mathrm{H}$ and ${ }^{13} \mathrm{C}$ NMR spectra were recorded on $400 \mathrm{MHz}$ Bruker Avance III FT-NMR spectrometer with tetramethylsilane (TMS) as internal standard. Chemical shifts $(\delta)$ are reported in parts per million (ppm) downfield of TMS. Elemental analysis was performed using Elementar Systeme (Vario EL III). Molecular mass was determined by electron impact (EI) method using GC-MS (Agilent GC-7890A, Mass-5975C).

General procedure for the synthesis of bridged esters $\mathbf{5}$. Aldehydes 9 were synthesized via formylation ${ }^{18,19}$ of anthracene by a known procedure. Aldehydes $9(16 \mathrm{mmol})$ were oxidized with $t$-butyl hydroperoxide $(1.92 \mathrm{~mL}$, $20 \mathrm{mmol}$ ) and $\mathrm{Se}(\mathrm{IV})$ oxide $(0.14 \mathrm{~g}, 1.25 \mathrm{mmol})(48-70 \mathrm{~h})$ in $t$-butanol at $75{ }^{\circ} \mathrm{C}$. Undissolved materials were filtered off and filtrate was evaporated. The residue thus obtained was dissolved in dichloromethane $(120 \mathrm{~mL})$ and stirred with $5 \mathrm{~N} \mathrm{HCl}(200 \mathrm{~mL})$ at room temperature for $4 \mathrm{~h}$. The aqueous and organic layers were separated and from the aqueous layer, the acids were extracted with dichloromethane. The organic solutions were collected and dried over anhydrous sodium sulfate and solvents were evaporated to obtain corresponding acids 10 (76-80\% yield, Table 1). Triethylamine $(1.40 \mathrm{~mL}, 10 \mathrm{mmol})$ was added to a solution of 10 (10 mmol) and cyanuric chloride $(1.84 \mathrm{~g}, 10 \mathrm{mmol})$ in acetone and stirred at room temperature for $1 \mathrm{~h}$ to get the corresponding acid chloride 12. Propargyl alcohol $(0.60 \mathrm{~mL}, 10 \mathrm{mmol})$ was added into it (one pot reaction) and the mixture was stirred for $4 \mathrm{~h}$. The products obtained were washed with sodium bicarbonate and extracted with dichloromethane. Esters $\mathbf{1 3}$ were purified by silica gel column chromatography using a mixture of hexane and dichloromethane as eluents. Pure products were obtained in 82-90\% yield. Anthracene derivatives appended with acetylinic substituents $13(5 \mathrm{mmol})$ were refluxed in $p$-xylene $(10 \mathrm{~mL})(48-64 \mathrm{~h})$ to generate corresponding barrelenes 5 that were purified by silica gel column chromatography using a mixture of hexane and dichloromethane as eluents (80-90\% yield).

Table 1. Amount of reactants taken in each step of the reactions and yields of intermediates $\mathbf{1 0}$ and $\mathbf{1 3}$

\begin{tabular}{|c|c|c|c|c|c|c|c|}
\hline \multicolumn{2}{|c|}{$\begin{array}{l}\text { Aldehydes 9, } \\
16 \mathrm{mmol}(\mathrm{g})\end{array}$} & \multicolumn{2}{|c|}{$\begin{array}{c}\text { Acids 10, } \\
10 \mathrm{mmol}(\mathrm{g})\end{array}$} & \multirow{2}{*}{$\begin{array}{c}\text { Yield \% } \\
78\end{array}$} & \multicolumn{2}{|c|}{$\begin{array}{l}\text { Targets 13, } \\
5 \mathrm{mmol}(\mathrm{g})\end{array}$} & \multirow{2}{*}{$\begin{array}{c}\text { Yield \% } \\
90\end{array}$} \\
\hline 9a) & 3.30 & 10a) & 2.20 & & 13a) & 1.30 & \\
\hline 9b) & 3.52 & 10b) & 2.36 & 76 & 13b) & 1.37 & 82 \\
\hline 9c) & 3.77 & 10c) & 2.52 & 79 & 13c) & 1.45 & 85 \\
\hline 9d) & 4.51 & 10d) & 2.98 & 80 & 13d) & 1.68 & 83 \\
\hline
\end{tabular}

General procedure for the synthesis of bridged sulfides 6 . Aldehydes 9 (16 mmol) dissolved in methanol, were reduced to corresponding alcohols 14 using sodium borohydride $(1.1 \mathrm{~g}, 30 \mathrm{mmol})$ in methanol. Alcohols 
$14(10 \mathrm{mmol})$ and two equivalents of thiourea $(1.5 \mathrm{~g}, 20 \mathrm{mmol})$ were dissolved in acetone $(25 \mathrm{~mL})$ and $5 \mathrm{~N} \mathrm{HCl}$ $(5 \mathrm{~mL})$ was added to it and stirred overnight. The precipitate formed was filtered and treated with sodium hydroxide $(10 \%, 30 \mathrm{~mL})$ solution and stirred at room temperature for $2 \mathrm{~h}$. Acidification with $5 \mathrm{~N} \mathrm{HCl}(25 \mathrm{~mL})$ yielded 16 in 87-95\% yield as shown in Table 2. To a solution of anthracenethiols 16 (5 mmol) dissolved in chloroform $(20 \mathrm{ml}), \mathrm{KOH}(0.20 \mathrm{~g}, 5 \mathrm{mmol})$ dissolved in methanol was added at $0{ }^{\circ} \mathrm{C}$ followed by propargyl bromide $(0.38 \mathrm{~mL}, 5 \mathrm{mmol})$ and stirred overnight. Reaction mixture was concentrated, washed with water and extracted with dichloromethane to obtain thioethers 17 in 75-85\% yields. Thioethers 17 were purified by silica gel column chromatography using a mixture of hexane and dichloromethane as eluents. IMDA reaction of 17 $(5 \mathrm{mmol})$ was effected by refluxing in $p$-xylene $(10 \mathrm{~mL})(5-10 \mathrm{~h})$ to obtain corresponding barrelenes 6 in $70-80$ $\%$ yields after recrystallization from suitable solvents.

Table 2. Amounts and yields of formation of intermediates 14, 16 and 17

\begin{tabular}{|c|c|c|c|c|c|c|c|c|}
\hline \multicolumn{2}{|c|}{$\begin{array}{l}\text { Alcohols 14, } \\
10 \mathrm{mmol}(\mathrm{g})\end{array}$} & \multirow{2}{*}{$\frac{\text { Yield \% }}{92}$} & \multicolumn{2}{|c|}{$\begin{array}{l}\text { Thiols 16, } \\
5 \mathrm{mmol}(\mathrm{g})\end{array}$} & \multirow{2}{*}{$\begin{array}{c}\text { Yield \% } \\
95\end{array}$} & \multicolumn{2}{|c|}{$\begin{array}{l}\text { Thioethers 17, } \\
5 \mathrm{mmol}(\mathrm{g})\end{array}$} & \multirow{2}{*}{$\frac{\text { Yield \% }}{83}$} \\
\hline 14a) & 2.08 & & 16a) & 1.12 & & 17a) & 1.38 & \\
\hline 14b) & 2.22 & 88 & 16b) & 1.19 & 92 & 17b) & 1.45 & 85 \\
\hline 14c) & 2.38 & 86 & 16c) & 1.27 & 87 & 17c) & 1.53 & 75 \\
\hline 14d) & 2.84 & 90 & 16d) & 1.50 & 90 & 17d) & 1.76 & 78 \\
\hline
\end{tabular}

Synthesis of tethered sulfone 7. Tethered barrelene 6 a $(200 \mathrm{mg}, 0.76 \mathrm{mmol})$ was dissolved in DMF (5 mL) and stirred with hydrogen peroxide $(30 \%, 2 \mathrm{~mL})$ and boric acid $(0.006 \mathrm{~g}, 0.1 \mathrm{mmol})$ for $12 \mathrm{~h}$ to obtain the corresponding sulfone 7 (78\%).

General procedure for the synthesis of tethered ethers 8. Aldehydes $9(16 \mathrm{mmol})$ were reduced to anthracene methanols 14 using sodium borohydride $(1.1 \mathrm{~g}, 30 \mathrm{mmol})$ dissolved in methanol. Anthracenemethanols $14(10 \mathrm{mmol})$ were converted to the corresponding sodium salts 18 by treating with sodium hydride $(0.48 \mathrm{~g}, 20 \mathrm{mmol})$ in THF. Propargyl bromide was added to it and stirred at room temperature for $2 \mathrm{~h}$ followed by refluxing in THF for $4 \mathrm{~h}$ to obtain 19 (75-85 \% yield, Table 3). Propargyl ethers 19 (5 mmol) were refluxed in $p$-xylene $(10 \mathrm{~mL})(12 \mathrm{~h}$ to $20 \mathrm{~h})$ to obtain the corresponding barrelenes $8(80-90 \%)$. The products were purified by silica gel column chromatography using a mixture of hexane and dichloromethane as eluents followed by recrystallization from suitable solvents and structures were confirmed by spectral and analytical data.

Table 3. Amounts and yields of formation of intermediate $\mathbf{1 9}$

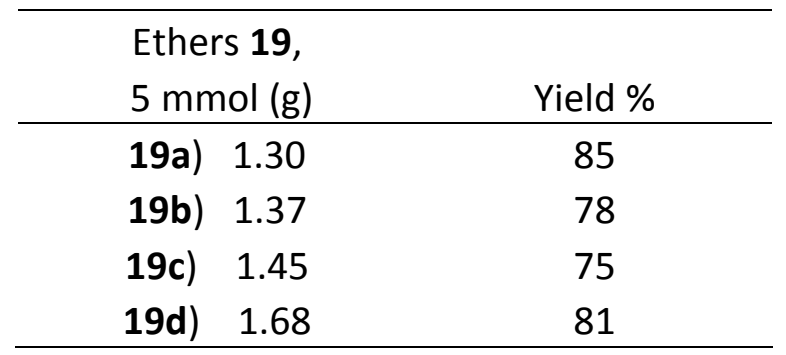

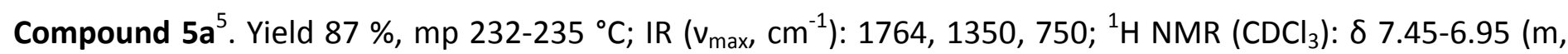
$8 \mathrm{H}), 6.75\left(\mathrm{dt}, 1 \mathrm{H}, J_{1} 6 \mathrm{~Hz}, J_{2} 2.4 \mathrm{~Hz}\right), 5.21(\mathrm{~d}, 1 \mathrm{H}, J 5.6 \mathrm{~Hz}) .4 .98(\mathrm{~d}, 2 \mathrm{H}, J 2.4 \mathrm{~Hz}) ;{ }^{13} \mathrm{C} \mathrm{NMR}\left(\mathrm{CDCl}_{3}\right): \delta 173.1$, 
147.0, 144.6, 142.5, 129.8, 125.5, 124.9, 123.5, 121.2, 68.2, 59.6, 52.2; MS: $m / z 260\left(M^{+}\right)$; Anal. Calcd. for $\mathrm{C}_{18} \mathrm{H}_{12} \mathrm{O}_{2}: \mathrm{C}: 83.06, \mathrm{H}: 4.65$. Found: $\mathrm{C}: 83.01, \mathrm{H}: 4.62$.

Compound 5b. Yield $82 \%, \mathrm{mp} 175-178{ }^{\circ} \mathrm{C}$; IR $\left(v_{\max }, \mathrm{cm}^{-1}\right): 1778,1350,750 ;{ }^{1} \mathrm{H} \mathrm{NMR}\left(\mathrm{CDCl}_{3}\right): \delta 7.52-7.01(\mathrm{~m}$, $8 \mathrm{H}), 6.43(\mathrm{t}, 1 \mathrm{H}, J 2.4 \mathrm{~Hz}), 5.03(\mathrm{~d}, 2 \mathrm{H}, J 2.4 \mathrm{~Hz}), 2.23(\mathrm{~s}, 3 \mathrm{H}) ;{ }^{13} \mathrm{C} \mathrm{NMR}\left(\mathrm{CDCl}_{3}\right): \delta 173.2,147.5,146.9,143.5$, 134.0, 125.2, 124.5, 120.8, 120.6, 68.0, 59.1, 51.3, 15.4; MS: m/z $274\left(M^{+}\right)$. Anal. Calcd. for $\mathrm{C}_{19} \mathrm{H}_{14} \mathrm{O}_{2}: \mathrm{C}: 83.19$, $\mathrm{H}:$ 5.14. Found: C: $83.10, \mathrm{H}: 5.12$.

Compound 5c. Yield $88 \%, \mathrm{mp} 200-202{ }^{\circ} \mathrm{C}$; IR $\left(v_{\max }, \mathrm{cm}^{-1}\right): 1769,1330,750 ;{ }^{1} \mathrm{H}$ NMR $\left(\mathrm{CDCl}_{3}\right): \delta 7.44-6.93(\mathrm{~m}$, $9 \mathrm{H}), 4.99$ (d, 2H, J $2 \mathrm{~Hz}), 3.94$ (s, 3H); ${ }^{13} \mathrm{C} \mathrm{NMR}\left(\mathrm{CDCl}_{3}\right): \delta 172.6,145.8,144.8,140.8,127.9,125.4,124.9,120.8$, 120.7, 88.5, 67.9, 57.9, 55.2; MS: $m / z 290\left(M^{+}\right)$; Anal. Calcd. for $\mathrm{C}_{19} \mathrm{H}_{14} \mathrm{O}_{3}$ : C: 78.61, H: 4.86. Found: C: 78.52 , H: 4.78 .

Compound 5d. Yield $85 \%, \mathrm{mp} 274-276{ }^{\circ} \mathrm{C}$; IR $\left(v_{\text {max }}, \mathrm{cm}^{-1}\right): 1778,1320,750 ;{ }^{1} \mathrm{H} \mathrm{NMR}\left(\mathrm{CDCl}_{3}\right): \delta 7.70-6.94(\mathrm{~m}$, $14 \mathrm{H}), 5.11$ (d, $2 \mathrm{H}, J 2.4 \mathrm{~Hz}) ;{ }^{13} \mathrm{C} \mathrm{NMR}\left(\mathrm{CDCl}_{3}\right): \delta 173.1,149.1,147.2,142.9,134.5,130.0,129.5,128.7,127.9$, 124.9, 124.9, 123.8, 121.0, 68.1, 61.3, 59.8; MS: $\mathrm{m} / \mathrm{z} 336\left(\mathrm{M}^{+}\right)$; Anal. Calcd. for $\mathrm{C}_{24} \mathrm{H}_{16} \mathrm{O}_{2}: \mathrm{C}: 85.69, \mathrm{H}:$ 4.79.Found: C: $85.61, \mathrm{H}: 4.70$.

Compound $6 \mathrm{a}^{5}$. Yield $80 \%, \mathrm{mp} 129-132{ }^{\circ} \mathrm{C}$; IR $\left(v_{\max }, \mathrm{cm}^{-1}\right): 1350,750 ;{ }^{1} \mathrm{H} N M R\left(\mathrm{CDCl}_{3}\right): \delta 7.30-6.95(\mathrm{~m}, 8 \mathrm{H})$, $6.66\left(\mathrm{dt}, 1 \mathrm{H}, J_{1} 6 \mathrm{~Hz}, J_{2} 1.6 \mathrm{~Hz}\right), 5.09(\mathrm{~d}, 1 \mathrm{H}, J 6 \mathrm{~Hz}), 3.98(\mathrm{~s}, 2 \mathrm{H}), 3.57(\mathrm{~d}, 2 \mathrm{H}, J 1.6 \mathrm{~Hz}) ;{ }^{13} \mathrm{C} \mathrm{NMR}\left(\mathrm{CDCl}_{3}\right): \delta 155.3$, 147.6, 145.8, 128.9, 124.6, 124.2, 122.9, 120.0, 64.2, 51.6, 33.4, 30.7; MS: $\mathrm{m} / \mathrm{z} 262\left(M^{+}\right)$; Anal. Calcd. for $\mathrm{C}_{18} \mathrm{H}_{14} \mathrm{~S}: \mathrm{C}: 82.40, \mathrm{H}: 5.38, \mathrm{~S}: 12.22$. Found: C: 82.34, H: 5.37, S: 12.29.

Compound 6b. Yield $74 \%$, mp 112-114 ${ }^{\circ} \mathrm{C}$; IR $\left(v_{\max }, \mathrm{cm}^{-1}\right): 2965,1350,750 ;{ }^{1} \mathrm{H} \mathrm{NMR}\left(\mathrm{CDCl}_{3}\right): \delta 7.29-7.00(\mathrm{~m}$, $8 \mathrm{H}), 6.29(\mathrm{t}, 1 \mathrm{H}, J 2 \mathrm{~Hz}), 3.98(\mathrm{~s}, 2 \mathrm{H}), 3.58(\mathrm{~d}, 2 \mathrm{H}, J 2 \mathrm{~Hz}), 2.16(\mathrm{~s}, 3 \mathrm{H}) ;{ }^{13} \mathrm{C} \mathrm{NMR}\left(\mathrm{CDCl}_{3}\right): \delta$ 156.0, 149.9, 146.8, 133.3, 124.3, 124.0, 120.0, 119.6, 63.6, 49.9, 33.5, 31.0, 15.7; MS: $m / z$ 276( $\left.M^{+}\right)$; Anal. Calcd. for $\mathrm{C}_{19} \mathrm{H}_{16} \mathrm{~S}: \mathrm{C}$ : 82.56, H: 5.83, S: 11.61 . Found: C: $82.51, \mathrm{H}: 5.87, \mathrm{~S}: 11.62$.

Compound 6c. Yield $78 \%, \mathrm{mp} 153-156{ }^{\circ} \mathrm{C}$; IR $\left(v_{\max }, \mathrm{cm}^{-1}\right): 2936,1350,750 ;{ }^{1} \mathrm{H} \mathrm{NMR}\left(\mathrm{CDCl}_{3}\right): \delta 7.39-6.91(\mathrm{~m}$, $8 \mathrm{H}), 6.79(\mathrm{t}, 1 \mathrm{H}, J 2 \mathrm{~Hz}), 3.90(\mathrm{~s}, 3 \mathrm{H}), 3.88(\mathrm{~s}, 2 \mathrm{H}), 3.54(\mathrm{~d}, 2 \mathrm{H}, J 2 \mathrm{~Hz}) ;{ }^{13} \mathrm{C} \mathrm{NMR}\left(\mathrm{CDCl}_{3}\right): \delta$ 153.1, 146.6, 143.2, 125.9, 123.5, 123.3, 118.9, 118.5, 86.2, 61.8, 53.9, 32.6, 29.8; MS: $m / z$ 292( $\left.M^{+}\right)$; Anal. Calcd. for $\mathrm{C}_{19} \mathrm{H}_{16} \mathrm{OS}: \mathrm{C}$ : 78.05, H: 5.52, S: 10.97. Found: C: 78.01, H: 5.44, S: 10.88.

Compound 6d. Yield $75 \%$, mp 218-221 ${ }^{\circ} \mathrm{C}$; IR $\left(v_{\max }, \mathrm{cm}^{-1}\right): 1350,750 ;{ }^{1} \mathrm{H} \mathrm{NMR}\left(\mathrm{CDCl}_{3}\right): \delta 7.71-6.89(\mathrm{~m}, 14 \mathrm{H})$, $4.06(\mathrm{~s}, 2 \mathrm{H}), 3.66$ (d, 2H, J $1.6 \mathrm{~Hz}) ;{ }^{13} \mathrm{C} \mathrm{NMR}\left(\mathrm{CDCl}_{3}\right):-\delta 157.4,150.1,146.1,135.5,130.2,128.6,128.5,127.5$, 124.3, 124.0, 123.1, 119.9, 63.8, 60.0, 33.9, 31.2; MS: $\mathrm{m} / z$ 338( $\left.\mathrm{M}^{+}\right)$; Anal. Calcd. for $\mathrm{C}_{24} \mathrm{H}_{18} \mathrm{~S}: \mathrm{C}: 85.17, \mathrm{H}: 5.36, \mathrm{~S}:$ 9.47. Found: C: $85.08, \mathrm{H}: 5.38, \mathrm{~S}: 9.54$.

Compound 7. Yield $78 \%, \mathrm{mp} 129-132{ }^{\circ} \mathrm{C} ; \mathrm{IR}\left(v_{\max }, \mathrm{cm}^{-1}\right): 1318,1300,750 ;{ }^{1} \mathrm{H} \mathrm{NMR}\left(\mathrm{CDCl}_{3}\right): \delta 7.26-6.87(\mathrm{~m}, 9 \mathrm{H})$, $5.09(\mathrm{~d}, 1 \mathrm{H}, \mathrm{J} 6 \mathrm{~Hz}), 4.10(\mathrm{~s}, 2 \mathrm{H}), 3.80-3.73(\mathrm{~m}, 2 \mathrm{H}) ;{ }^{13} \mathrm{C} \mathrm{NMR}\left(\mathrm{CDCl}_{3}\right): \delta$ 148.3, 145.6, 144.3, 134.4, 133.3, 119.4, 119.0, 118.7, 58.48, 53.9, 50.5, 50.2; MS: $m / z$ 294( $\left.M^{+}\right)$; Anal. Calcd. for $\mathrm{C}_{18} \mathrm{H}_{14} \mathrm{O}_{2} \mathrm{~S}: \mathrm{C}: 73.44, \mathrm{H}: 4.79, \mathrm{~S}: 10.89$. Found: C: 73.41, H: 4.78, S: 10.91.

Compound $8 \mathrm{a}^{5}$. Yield $90 \%, \mathrm{mp} 198-200{ }^{\circ} \mathrm{C}$; IR $\left(v_{\max }, \mathrm{cm}^{-1}\right): 2880,1350,750 ;{ }^{1} \mathrm{H}_{\mathrm{NMR}}\left(\mathrm{CDCl}_{3}\right): \delta 7.29-6.97(\mathrm{~m}$, $8 \mathrm{H}), 6.61\left(\mathrm{dt}, 1 \mathrm{H}, J_{1} 6 \mathrm{~Hz}, J_{2} 2 \mathrm{~Hz}\right), 5.16(\mathrm{~d}, 1 \mathrm{H}, J 6 \mathrm{~Hz}), 4.99(\mathrm{~s}, 2 \mathrm{H}), 4.41(\mathrm{~d}, 2 \mathrm{H}, J 2 \mathrm{~Hz}) ;{ }^{13} \mathrm{C} \mathrm{NMR}\left(\mathrm{CDCl}_{3}\right): \delta 154.9$, 147.2, 145.1, 125.7, 124.6, 124.3, 123.0, 120.0, 67.5, 66.4, 62.1, 52.0; MS: $\mathrm{m} / \mathrm{z} 246\left(M^{+}\right)$; Anal. Calcd. for $\mathrm{C}_{18} \mathrm{H}_{14} \mathrm{O}: \mathrm{C}: 87.78, \mathrm{H}: 5.73$. Found: $\mathrm{C}: 87.76, \mathrm{H}: 5.7$.

Compound 8b. Yield 81 \%, mp 139-142 ${ }^{\circ} \mathrm{C}$; IR $\left(v_{\max }, \mathrm{cm}^{-1}\right): 2925,1350,750 ;{ }^{1} \mathrm{H}$ NMR $\left(\mathrm{CDCl}_{3}\right): \delta 7.32-6.99(\mathrm{~m}$, $8 \mathrm{H}), 6.25(\mathrm{t}, 1 \mathrm{H}, J 2 \mathrm{~Hz}), 5.00(\mathrm{~s}, 2 \mathrm{H}), 4.42(\mathrm{~d}, 2 \mathrm{H}, J 2 \mathrm{~Hz}), 2.19(\mathrm{~s}, 3 \mathrm{H}) ;{ }^{13} \mathrm{C} \mathrm{NMR}\left(\mathrm{CDCl}_{3}\right): \delta$ 154.5, 148.5, 145.1, $128.9,123.4,123.0,119.0,118.6,66.53,65.5,60.5,49.4,14.7 ; \mathrm{MS}: \mathrm{m} / z$ 260( $\left.M^{+}\right)$; Anal. Calcd. for $\mathrm{C}_{19} \mathrm{H}_{16} \mathrm{O}: \mathrm{C}$ : 87.66, H: 6.19. Found: C: 87.62, H: 6.17.

Compound 8c. Yield $85 \%$, mp 155-158 ${ }^{\circ} \mathrm{C}$; IR $\left(v_{\max }, \mathrm{cm}^{-1}\right): 2940,1350,750 ;{ }^{1} \mathrm{H} \mathrm{NMR}\left(\mathrm{CDCl}_{3}\right): \delta 7.49-6.98(\mathrm{~m}$, $8 \mathrm{H}), 6.83(\mathrm{t}, 1 \mathrm{H}, J 3 \mathrm{~Hz}), 5.00(\mathrm{~s}, 2 \mathrm{H}), 4.56(\mathrm{~d}, 2 \mathrm{H}, J 3 \mathrm{~Hz}), 4.00(\mathrm{~s}, 3 \mathrm{H}) ;{ }^{13} \mathrm{C} \mathrm{NMR}\left(\mathrm{CDCl}_{3}\right): \delta$ 153.7, 147.3, 143.6, 
124.6, 124.3, 123.7, 120.1, 119.6, 87.9, 67.6, 66.6, 60.6, 55.0; MS: $m / z$ 276(M $\left.{ }^{+}\right)$. Anal. Calcd. for $\mathrm{C}_{19} \mathrm{H}_{16} \mathrm{O}_{2}: \mathrm{C}$ 82.58, H: 5.84. Found: C: 82.49, H: 5.84.

Compound 8d. Yield $87 \%, \mathrm{mp} 233-236{ }^{\circ} \mathrm{C}$; IR $\left(v_{\max }, \mathrm{cm}^{-1}\right): 2863,1350,750 ;{ }^{1} \mathrm{H} \mathrm{NMR}\left(\mathrm{CDCl}_{3}\right): \delta 7.24-7.35(\mathrm{~m}$,

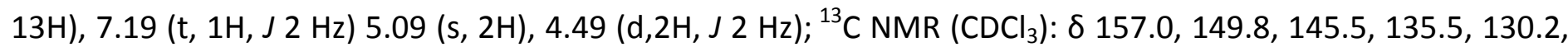
128.5, 127.5, 125.4, 124.3, 124.0, 123.2, 119.8, 67.8, 66.7, 61.8, 60.5; MS: $\mathrm{m} / \mathrm{z} 322\left(M^{+}\right)$; Anal. Calcd. for $\mathrm{C}_{24} \mathrm{H}_{18} \mathrm{O}: \mathrm{C}: 89.41, \mathrm{H}: 5.63$. Found: $\mathrm{C}: 89.36, \mathrm{H}: 5.61$.

\section{Acknowledgements}

We thank CSIR, DST, Govt. of India for funding and Sophisticated Test and Instrumentation Centre (STIC)CUSAT for the spectral and analytical data.

\section{References}

1. Zimmerman, H. E.; Paufler, R. M. J. Am. Chem. Soc. 1960, 82, 1514.

https://pubs.acs.org/doi/abs/10.1021/ja01491a071

2. Cristol, S. J.; Bly, R. K. J. Am. Chem. Soc. 1960, 82, 6155.

http://pubs.acs.org/doi/pdf/10.1021/ja01508a046

3. Miller, R. G.; Styles, M. J. Am. Chem. Soc. 1963, 85, 1798.

http://pubs.acs.org/doi/pdf/10.1021/ja00895a023

4. Figeys, H. P.; Dralants, A. Tetrahedron 1972, 28, 3031. https://www.sciencedirect.com/science/article/pii/0040402072800188/pdf?md5=cf85540c8c3d1cbc5083f 86f37f615a3\&isDTMRedir=Y\&pid=1-s2.0-0040402072800188-main.pdf\& valck=1

5. Ciganek, E. J. Org. Chem. 1980, 45, 1497. http://pubs.acs.org/doi/pdf/10.1021/jo01296a031

6. Diels, O.; Alder, K. Justus Liebigs Ann. Chem. 1928, 460, 98.

https://doi.org/10.1002\%2Fjlac.19284600106

7. Roush, W. R.; Kageyama, M.; Riva, R.; Brown, B. B.; Warmus, J. S.; Moriarty, K. J. J. Org. Chem. 1991, 56, 1192.

http://pubs.acs.org/doi/pdf/10.1021/jo00003a049

8. Meek, J. S.; Dann, J. R. J. Org. Chem. 1956, 21, 968. http://pubs.acs.org/doi/pdf/10.1021/ja01495a043

9. Meek, J. S.; Wilgus, D. R.; Dann, J. R. J. Am. Chem. Soc. 1960, 82, 2566. http://pubs.acs.org/doi/pdf/10.1021/ja01495a043

10. Zimmerman, H. E.; Grunewald, G. L. J. Am. Chem. Soc 1966, 88, 183.

http://pubs.acs.org/doi/pdf/10.1021/ja00953a045

11. Zimmerman, H. E.; Binkley, R. W.; Givens, R. S.; Sherwin, M. A. J. Am. Chem. Soc 1967, 89, 3932. http://pubs.acs.org/doi/pdf/10.1021/ja00991a064

12. Hixson, S. S.; Mariano, P. S.; Zimmerman, H. E. Chem. Rev. 1973, 73, 531.

http://pubs.acs.org/doi/pdf/10.1021/cr60285a005

13. Ramaiah, D.; Sajimon, M. C.; Joseph, J.; George, M. V. Chem. Soc. Rev. 2005, 34, 48. http://pubs.rsc.org/en/content/articlehtml/2005/cs/b300843f

14. Ganschow, M.; Koser, S,; Hahn, S.; Rominger, F.; Freudenberg, J.; Bunz, U. Chem. Eur. J. 2017, $23,4415$. http://onlinelibrary.wiley.com/doi/10.1002/chem.201605820/full 
15. Ishii, A.; Shibata, M.; Ebina, R.; Nakata, N. Eur. J. Org. Chem. 2018, 1011.

https://onlinelibrary.wiley.com/doi/abs/10.1002/ejoc.201701616

16. Ishii, A.; Shibata, M.; Nakata, N. Bull. Chem. Soc. Jpn. 2016, 89, 1470.

http://www.journal.csj.jp/doi/abs/10.1246/bcsj.20160287

17. Khalil, A. M.; Berghot, M. A.; Gouda, M. A. Eur. J. Med. Chem. 2009, 44, 4434.

https://www.sciencedirect.com/science/article/pii/S0223523409003286

18. Vetter, S. Synth.Commun. 1998, 28, 3219.

http://www.tandfonline.com/doi/pdf/10.1080/00397919808004425

19. Lane, B. S.; Burgess, K. Chem. Rev. 2003, 103, 2457.

http://pubs.acs.org/doi/abs/10.1021/cr020471z 